\title{
ARABIC ONLINE HANDWRITING RECOGNITION USING NEURAL NETWORK
}

\author{
Abdelkarim Mars $^{1}$ and Georges Antoniadis ${ }^{2}$ \\ ${ }^{1}$ Laboratory LIDILEM, Alpes University, Grenoble, French \\ ${ }^{2}$ Laboratory LIDILEM, Alpes University, Grenoble, French
}

\begin{abstract}
This article presents the development of an Arabic online handwriting recognition system. To develop our system, we have chosen the neural network approach. It offers solutions for most of the difficulties linked to Arabic script recognition. We test the approach with our collected databases. This system shows a good result and it has a high accuracy (98.50\% for characters, $96.90 \%$ for words).
\end{abstract}

\section{KEYWORDS}

Neural Network, Handwriting recognition, Online, Arabic Script

\section{INTRODUCTION}

In the last ten years, considerable progress has been made in the field of handwriting recognition. This progress is due to the many work in this field and also the availability of international standards databases for handwriting that allowed the researchers to report in a credible way the performance of their approaches in this field, with the possibility of comparing them with other approaches which they use the same bases.

The Arabic language was not so lucky, unlike Latin, it is still on the level of the research and experimentation [1], that is to say that the problem remains an open challenge for researchers.

The Arabic script is cursive by nature, it poses many problems for automatic handwriting recognition systems. The most difficult problem in the design of a handwriting recognition system is the segmentation of handwritten words for their recognition, which requires much time consuming and calculation. On the other hand, the local information are somewhat neglected in systems based on a global analysis which can significantly reduce performance [2].

To remedy these problems, neural network approaches have been proposed for the recognition of handwritten Arabic words . Such a system requires the consideration of a large number of variabilities writing.

In this context, it is appropriate to ask the following question: How can we use neural network on the Arabic HWR? 
The paper will be organized as follows: Section 2 presents the related work; Section 3 describes the Arabic script; Section 4 provides the proposed neural network method; Section 5 and 6 describes technical details about collection of the database and the use of neural network on Arabic HWR; Section 7 reports the results; and section 8 concludes the paper and provides possible future works.

\section{BACKROUND}

The handwriting recognition is a data processing which aims to translate a written text in a digitally encoded text by the computer. There are two types of handwriting; printed characters and cursive handwriting. These writing varieties affect the quality of the recognition system and they cause many problems during the segmentation step. The handwriting recognition can be classified into two areas:

- Online Recognition: used with the digital pen or the touch interface.

- offline Recognition: generally we use this type of recognition of the scanned writing, writing on photos, verifying signatures on bank checks.

The first work on the handwriting recognition has been made in the fifties and sixties. Because of the poor performance of these systems, little research on handwriting recognition took place during the eighties. The problem of handwriting recognition was considered very difficult to solve [3]. Although there are many online handwriting recognition applications, the technology is not yet fully mature. There are still some improvements can still be made to handwriting recognition systems.

There is some research on online Arabic handwriting recognition, [4] suggest a method for handwriting character recognition drawn on a graphic tablet. In addition, [5] used a method based on confused matrices used to prune the nodes which cause the error (error causing). [6] proposed a new structural approach for recognizing Arabic handwritten digits based on primitives extraction. [7] proposed a system to recognize cursive handwriting based on rule-based method to perform segmentation and recognition portions of text in a cursive handwritten document by using dynamic programming. [8] developed an Arabic handwriting recognition system based on an arbitrary segmentation algorithm followed by segmenting improvement consecutive common connections and segmentation of the point locating.

The handwriting recognition engine uses the training data to recognize the fundamental individual units. In the recognition phase, the system will compare the test pattern with each reference class that represents the words of the lexicon and measuring a similarity score between the test pattern and the reference class. The similarity score must decide which model have the best fits the unknown form. The implementation of this recognition module in recognition system requires certain approaches such as decision tree, dynamic programming [9], hidden Markov model [10], neural network, k-nearest neighbor and other combinations of different approaches [11].

\section{ARABIC SCRIPT}

The Arabic writing is cursive and consonantal, it is composed by 28 letters, 12 special letters $(\downarrow$,

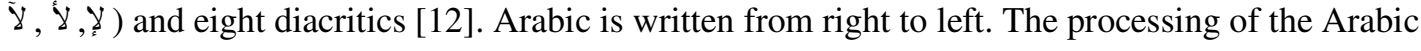


language is a difficult task, especially in the field of automatic handwriting recognition for many reasons:

- Arabic letters change their shapes depending on their position in the word; isolated, initial, middle, end.

Table 1. Change of the shape of a letter according to its position, example of variation of the letter $\varepsilon$ "Ayn".

\begin{tabular}{|l|l|l|l|}
\hline Isolated & Initial & Middle & end \\
\hline$\varepsilon$ & $\mathcal{L}$ & $\boldsymbol{c}$ & $\boldsymbol{\varepsilon}$ \\
\hline
\end{tabular}

- Many letters contain dots and dashes such as the letter Alif Mad "I". They are added by the writer at the end of handwritten letter.

- The style of writing in Arabic handwriting varies from one writer to another.

- An Arabic word must be written recursively and each characters must be connected to another one [13].

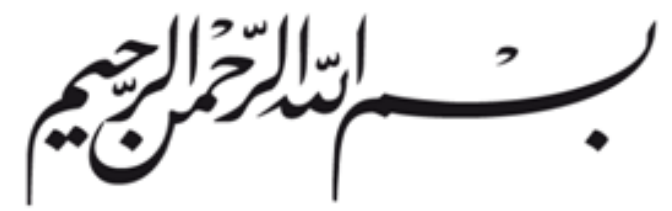

Figure 1. Connectivity between Arabic characters.

- Some Arabic words contain ligatures, as shown in the figure below.

Table 2. Arabic Ligature Lam Alif.

\begin{tabular}{|l|l|}
\hline corroct & $V \leftarrow I+J$ \\
\hline neorrect & $U \leftarrow I+J$ \\
\hline
\end{tabular}

\section{NEURAL NETWORK APPROACH}

There are many methods to train and model a handwriting recognition system. Among the existing methods, we mention the neural networks, hidden Markov model (HMM), the neighbor k-nearest, expert systems and other techniques. These methods can be divided into two main classes:

Syntax: the class that involves describing the character shapes in an abstract way.

Statistics: where the system learns from the data directly, without explicitly specifying the system knowledge structure. 
Neural networks have been used in the handwriting recognition system successfully. However, compared to other approaches, they require more calculations [14]. Neural networks is a static classifier that requires vector functions with fixed size. Due to these properties, the neural network is considered a very efficient approach to the recognition of characters and graphemes.

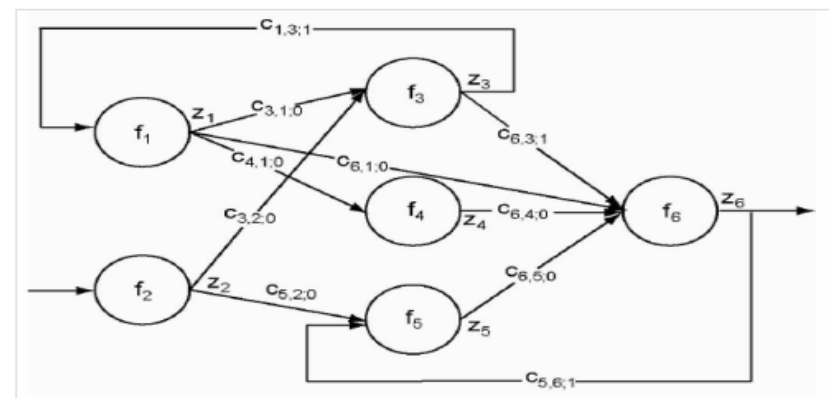

Figure 2. Neural network represented by a directed graph..

A neural network is composed of two parts, one is called Time Delay Neural Networks (TDNN) and the second one is called multi-layer perceptron (MLP). A MLP is composed by three layers: input layer, hidden layers and an output layer. Each neuron is connected to all the other neurons of the lower layer to build a network called a fully connected network [15].

\section{Creation an HWR database}

To train a handwriting recognition engine we need a database of ink containing reference information regarding the shape of each character or each word. These databases are very important to validate our system and our approach based on neural network to test the performance of our system.

To be generic and able to interpret as many variations as possible, the writers on collection step should be diverse as possible. For the Arabic writing, many databases have been produced, but not accessible to the public. In fact, existing ink databases was private and contains small dictionaries with limited vocabulary. Actually, there is no robust database developed for an Arabic handwriting recognition system.

View the existing databases quality and view the incompatibility (in size, diversity of writers, content, licence) of these bases to our needs, we should building our own databases.

In this step, we have to prepare 100 forms containing characters and words to recopy by writers. Each form consists of three pages contain the set of numbers and words. Therefore, we should prepare data to generate 100 forms ( 300 pages). The goal is to use 70 forms to train of our system and 30 forms for the test.

Characters: the first part of the form is composed of all the characters of the language; the alphabet that contains all the letters of a language (uppercase and lowercase), numbers that contain all of the numbers used in the language and symbols such as mathematical signs $(+,-, *)$, punctuation, symbols often used in writing (@, \&, \#, \$).

The words: they are diversified words taken from Aljazeera website. 
Once forms was generated and verified, the next step is to print in Anoto paper the forms. We need 100 native Arabic writers to filling out forms. In addition, we need digital pens that are necessary to recover the ink at the end of this step. In our collection, we used digital pen Nokia SU-1B. The spatial resolution of this pen is 677 dots per inch (dpi) and it have a sampling rate of 100 points per second $(100 \mathrm{~Hz})$.

\section{RECOGNITION PROCESS}

The first step in a recognition process, the engine takes as input an ink from an online equipment. To transform this into digital characters, several treatments are performed by the system. Among these components, mention the module that handles the pre-processing and normalization. Once the ink is pre-processed, another module extracts the necessary characteristics. These are used after extraction by the classification module based on neural network to give a hypothesis of character.

- Pre-processing and normalization: This process of handwriting recognition is necessary and crucial to achieve a better recognition rate. Generally, before recognition, the acquired inks are usually pre-treated [16]. Therefore, at this step the main objective of the pre-processing is to reduce noise on the databases, to eliminate trembling and physical imperfections while writing[5].

- Detecting reference lines: In a process of word recognition, reference lines provide important information for systems. It allows the normalization of the writing size and it helps the system to find the geometric features related to the orientation of the letters in the word.

- Delayed strokes handling: In Arabic writing, delayed strokes are written below or above the word and can appear before, after, or as a part of the words compared to the horizontal axis. Many researchers consider that the delayed strokes add more complexity to the process of online handwriting recognition and should be completely removed from the ink in the pre-treatment step [17]. To correct this problem due to delayed strokes, we develop a module that adds a stroke of connection to attach delayed stroke to the word.

- Features extraction : The main objective of feature extraction is to reduce the input pattern by extracting and calculating the characteristics or the parameters of the most relevant input signal (ink) to achieve the best classification model [18].

In our case, seven characteristics values were extracted for each item resulting. The characteristic values for each point $\mathrm{x}(\mathrm{n}), \mathrm{y}(\mathrm{n})$ are:

i) Normalized $x(n)$ between -1 and 1 .

ii) Normalized y (n) between -1 and 1 .

iii) The cosine of the angle made by the line between the point $x(n+1), y(n+1)$ and the point $\mathrm{x}(\mathrm{n}-1), \mathrm{y}(\mathrm{n}-1)$ and the $\mathrm{x}$-axis.

iv) The sinus of the angle made by the line between the point $x(n+1), y(n+1)$ and the point $\mathrm{x}(\mathrm{n}-1), \mathrm{y}(\mathrm{n}-1)$ and the $\mathrm{x}$-axis.

v) The cosine of the angle of curvature between the points $x(n+2), y(n+2)$ and the point $x(n-2), y(n-2)$ to $x(n), y(n)$.

vi) The sinus of the angle of curvature between the points $x(n+2), y(n+2)$ and the point $\mathrm{x}(\mathrm{n}-2), \mathrm{y}(\mathrm{n}-2)$ to $\mathrm{x}(\mathrm{n}), \mathrm{y}(\mathrm{n})$.

vii) Fixed the binary value 1 when the pen is down, or -1 when the pen is up.

The characteristics (iii) and (iv) provide direction information and characteristics (v) and

(vi) provide curvature information. The Figure shows in more detail, the four related 
characteristics curves in mode (iii), (iv), (v) and (vi). For recognition, the characteristic values for a single character are used.

- Segmentation: Segmentation refers to the various operations to be performed by the recognition system to obtain significant base units (stroke, grapheme, character, etc.) that the recognition algorithm can handle. This phase generally consists of two levels. The first level is the entire text and focuses on the detection line [19]. At the second level, the module focuses on segmentation into small individual units such as strokes, graphemes or characters. This is among the most difficult in the process of handwriting recognition. The figure below shows the maximum segmentation of the Arabic word "Alhob".

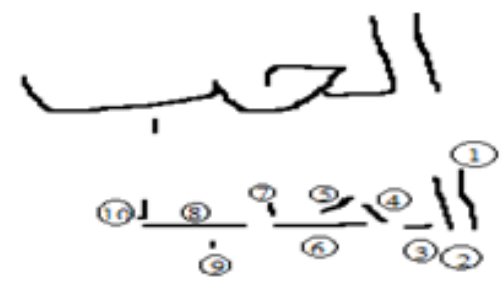

Figure 3. Example Maximum segmentation of the word "Alhob".

- Training and recognition : For our handwriting recognition system, we mainly use the convolution neural network, mainly TDNN [20]. After the phase of the segmentation and feature extraction, we obtain a lattice in the output of the TDNN. Then, from this output, we calculate the probability of each word in the dictionary. Words are represented on the TDNN as a sequence of characters and each character is modeled by one or more states in the neural networks. Therefore, the TDNN can be considered as a hybrid recognition device that combines the features of neural networks. Each state of the character (isolated, at the beginning, at the middle, at the end) was represented by a neuron in the input layer. Usually the score of a character is calculated by finding the optimal alignment path through its states and adding activations in this direction. Similarly, the score of a word is calculated by finding the optimal alignment path through the states of characters in the word. Therefore, the final score is calculated by adding all activated states in this direction [21].

We have developed this algorithm for learning TDNN:

Definition and allocation of TDNN

Initialization of all weights

Construction of a standardized training base

For each example e from learning base

Resampling of Example e

Standardization of example e

Extraction and saving all the features of the example e

Saving the label of example $e$

While the stop condition is not satisfied

For each example e from to the learning base

Propagation of example e

Calculate the local error

If the criterion of the local error criterion of the example e is not satisfied 
International Journal of Artificial Intelligence and Applications (IJAIA), Vol. 7, No. 5, September 2016

\section{Back propagation of error \\ Checking the stop condition \\ End while \\ // Calculate the criterion}

For each example e from the learning base

Propagation of example e

Calculate the local error

Calculating the cumulative error

To calculate the errors we use the following function:

$$
E_{C E}=-\sum_{j}\left[d_{j} \log \left(y_{j}\right)+\left(1-d_{j}\right) \log \left(1-y_{j}\right)\right]
$$

\section{EVALUATION OF THE RECOGNITION SYSTEM}

In this section, we present results that correspond to the evaluation of our system of Arabic handwriting recognition. This evaluation is made on the database of test resulting from the collection. We used the correct recognition rate to evaluate the performance of our system. In addition, we will evaluate our system compared to MyScript.

MyScript is a software for the handwriting recognition developed by Vision Object. This is a paid system, but the company offers to developers the opportunity to test their recognition engine via the Cloud (online remote server) for a period of three months. We choose this software because it is functional for Arabic language; it gives good results and it is used by Samsung in their smartphones, so we have the opportunity to compare these results with ours. Against by, there is no information on the algorithms and approaches used by this system.

\subsection{Recognition test}

At this stage, we will evaluate our recognition system by comparing it with MyScript system. We will evaluate both systems using the same basis of tests.

Table 3. Comparative table of results.

\begin{tabular}{|l|l|l|l|}
\hline Data & Size of database test & Accuracy of our system & Accuracy of MyScript \\
\hline Characters & 6090 & 98.50 & 90.30 \\
\hline Words & 1080 & 96.90 & 88.90 \\
\hline
\end{tabular}

This table shows the accuracy of our system compared to the Myscript system using testing databases. We can see that our results are effective because we can know almost the characters. During the recognition phase, our system is almost in real-time. However, the accuracy of MyScript system is acceptable, but it is not effective in the case of recognition of Arabic sentences or Arabic texts.

The comparison of our system with that of MyScript proves that our results are classified. However, our system generates some recognition errors for Arabic language. 


\subsection{Recognition errors}

Error analysis of unrecognized Arabic words shows these errors:

- Errors associated with diacritics: during the segmentation phase, our system has difficulty to segment words that do not exist in the training databases. For example, words written with diacritical Chadda "" and those who are written with the letter Alif Madda "I", etc.

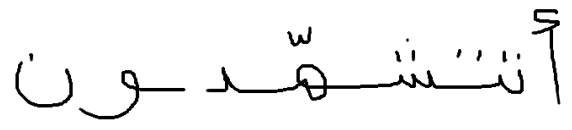

Figure 4. Sample written with diacritical Chaddah.

- Errors related to the overwriting: in the Arabic language, many people write the words superimposed many letters. Therefore, our system generates errors during the segmentation of superimposed characters.

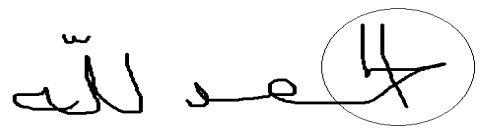

Figure 5. Sample with a superposition of the first three letters.

- Errors associated with deleting some characters at the end of the word: analyzing unrecognized words, we noticed that some writers do not write the entire words they write just the first characters after they make a dash for the rest of the characters.

-

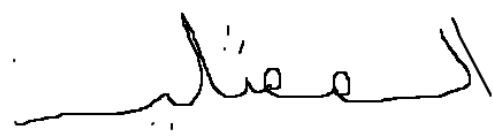

Figure 6. Sample of word " المثلي_ـ" with letters dropped at the end.

\section{Conclusions}

We proposed in this article an on-line Arabic handwriting recognition system based on neural networks approach. We suggest a method bases on TDNN classifier and multi-layer perceptron, which allow an effective results and a high accuracy compared to MyScript system. In addition, a neural networks approach allows us to reduce the computational complexity by exploiting the redundancy of the scanning window signal.

\section{REFERENCES}

[1] Essoukhri, Ben Amara, (2002) "Problématique et orientations en reconnaissance de l'écriture arabe", Colloque International Francophone sur l'Ecrit et le Document, pp.1.-10, Hammamet, Tunisie, Octobre 2002

[2] Essoukhri, Ben Amara, A, Belaïd. \& A, Ellouze., (2000), "Utilisation des modèles markoviens en reconnaissance de l'écriture arabe: Etat de l'art", CIFED'2000, Colloque International Francophone sur l'Ecrit et le Document, pp.181-191, Lyon, France, 2000. 
[3] G, Lorette., (2013), "Handwriting Recognition or Reading Situation". At the Dawn Of The 3rd Millenium", in Advances in Handwriting Recognition, World Scientific Publications, pp. 3-13.

[4] M, El-Wakil. \& A, Shoukry, (1989), "On-line recognition of handwritten isolated arabic characters". Pattern Recognition 22, 97-105 1989.

[5] N, Mezghani. A, Mitiche and M, Cheriet (2002) "On-line recognition of handwritten arabic characters using a kohonen neural network, in: Frontiers in Handwriting Recognition”, 2002. Proceedings. Eighth International Workshop on, IEEE. pp. 490-495.

[6] A, T, Al-Taani (2005) "An efficient feature extraction algorithm for the recognition of handwritten arabic digits". International journal of computational intelligence 2, 107-1112005.

[7] R, I, Elanwar. M,A, Rashwan \& S, A, Mashali (2007) "Simultaneous segmentation and recognition of arabic characters in an unconstrained on-line cursive handwritten document", in: Proceedings of world academy of science, engineering and technology, pp. 288-291 2007.

[8] K, Daifallah. N, Zarka \& H, Jamous (2009) "Recognition-based segmentation algorithm for on-line arabic handwriting”, in: Document Analysis and Recognition, 2009. ICDAR'09. 10th International Conference on, IEEE. pp. 886-890 2009.

[9] R, I, Elanwar. M, A, Rashwan.\& S, A, Mashali (2007). "Simultaneous segmentation and recognition of Arabic characters in an unconstrained on-line cursive handwritten document". Proceedings of World Academy of Science, Engineering and Technology (WASET), International conference on Machine learning and Pattern Recognition MLPR2007, vol. 23, pp. 288-291, Germany (2007).

[10] H, Boubaker. A, Chaabouni. M, Kherallah. A, M, Alimi \& H, El Abed (2010). "Fuzzy segmentation and graphemes modeling for online Arabic handwriting recognition". Proceedings of ICFHR 2010, pp. 695-700 (2010).

[11] R, Halavati. M, Jamzad. \& M, Soleymani (2005) "A novel approach to Persian online hand writing recognition". Trans. Eng. Comput. Technol. 6, 232-236.

[12] F, Bouslama \& A, Amin (1998) "Pen-based recognition system of Arabic character utilizing structural and fuzzy techniques". In: Proceedings of Second International Conference on Knowledge-Based Intelligent Electronic Systems, pp. 76-85 (1998).

[13] A, Mars. \& G, Antoniadis (2015) "Handwriting recognition system for Arabic language learning" WCITCA'2014 World Congress on Information Technology and Computer Application, HAMMAMET 2015 - International Journal N\&N Global technology.

[14] A, H, Ganapathiraju.\& J, Picone (2004) . "Applications of support vector machines to speech recognition". IEEE Transactions on Signal Processing, 52, 2348 - 2355.

[15] J, S, Bridle (1990) "Training Stochastic Model Recognition Algorithms as Networks Can Lead to Maximum Mutual Information Estimation of Parameters", in Advances in Neural Information Processing Sys-tems 2, D.S. Touretzky, ed., pages 211-21 7, 1990.

[16] D, Kim, (1999), Normalization Methods for Input and Output Vectors in Backpropagation Neural Networks, International Journal of Computer Mathematics, Vol. 71, No. 2, 161-171.

[17] N, Mezghani. A, Mitiche \& M, Cheriet (2008) "Bayes classification of online Arabic characters by Gibbs modeling of class conditional densities". IEEE Trans. Pattern Anal. Mach. Intell. 30(7), 112111.

[18] N, Intrator, (1992) "Feature Extraction Using an Unsupervised Neural Network", Neural Computation, vol. 4, no. 1, pp. 98-107, 1992

[19] S, Izadi. M, Haji and C,Y, Suen, (2008), A new segmentation algorithm for online handwritten word recognition in Persian script, 11th International Conference on Frontiers in Handwriting Recognition (CFHR 2008), Montreal, Canada, 2008, 598-603.

[20] V, Peddinti. D, Povey and S, Khudanpur, (2015), "A Time Delay Neural Network Architecture for Efficient Modeling of Long Temporal Contexts", Proceedings of Interspeech.

[21] A, Mars. \& G, Antoniadis (2015) "Handwriting recognition system for Arabic language learning". International Journal of Engineering and Advanced Technology Studies Vol.3, No.7, pp.55-63, September 2015. 